\title{
Novel Hexagonal Dual-Mode Substrate Integrated Waveguide Filter with Source-Load Coupling
}

\author{
Ziqiang Xu, Gen Zhang, Hong Xia, and Meijuan Xu \\ Research Institute of Electronic Science and Technology, University of Electronic Science and Technology of China, \\ Chengdu 611731, China \\ Correspondence should be addressed to Ziqiang Xu; nanterxu@uestc.edu.cn
}

Received 19 August 2013; Accepted 16 October 2013; Published 22 April 2014

Academic Editors: G. De Mey and G. Priebe

Copyright (C) 2014 Ziqiang Xu et al. This is an open access article distributed under the Creative Commons Attribution License, which permits unrestricted use, distribution, and reproduction in any medium, provided the original work is properly cited.

Hexagonal dual-mode cavity and its application to substrate integrated waveguide (SIW) filter are presented. The hexagonal SIW resonator which can combine flexibility of rectangular cavity and performance of circular cavity is convenient for dual-mode bandpass filters design. By introducing coupling between source and load, the filter not only has good selectivity due to two controllable transmission zeros, but also has a small size by the virtue of its single-cavity structure. A demonstration filter with a center frequency of $10 \mathrm{GHz}$ and a $3 \mathrm{~dB}$ fractional bandwidth of $4 \%$ is designed and fabricated to validate the proposed structure. Measured results are in good agreement with simulated ones.

\section{Introduction}

Dual-mode cavity bandpass filters have been widely used in the development of various wireless communication systems. The metal waveguide dual-mode filters have excellent performance owing to their high $Q$ factor and power-handling capability. However, they cannot be easily integrated with microwave planar circuits $[1,2]$. Recently the substrate integrated waveguide (SIW), which is synthesized in a planar substrate with arrays of metallic via, provides a lowprofile, low-cost, and low-weight scheme while maintaining high performance [3-5]. Particularly, the application of SIW technology makes the implement of dual-mode cavity filters with compact size and easy integration possible [6].

On the other hand, filters with multiple transmission zeros $(T Z s)$ are required to meet the increasing demands of modern communication systems in regards to compact size and high selectivity. Commonly, no more than one TZ can be obtained in a conventional single-cavity dual-mode filter. In order to generate more $T Z s$, many approaches have been proposed to design dual-mode SIW filters. One approach is cascading two adjacent dual-mode rectangular cavities to generate up to two TZs in stop band [7]. Similarly, a dual-mode filter using two connecting circular cavities with two TZs is introduced in [8]. However, their physical sizes will become larger because of cascaded structures. Another approach can be fulfilled by marshaling the effect of sourceload coupling in single cavity. By adding a direct signal path between the source and the load, $N$ finite transmission zeros can be generated. In [9], a dual-mode filter using a nonresonating node with indirect source-load coupling is proposed, and two TZs are obtained in such a single-cavity filter.

Ordinarily, conventional dual-mode SIW filers are always built based on rectangular and circular cavities. In our previous work, a novel hexagonal resonator using SIW technology and its applications to trisection filters are proposed in [10]. The hexagonal SIW cavity can combine flexibility of rectangular cavities and performances of circular cavities. Meanwhile, as any of the six sides of a hexagonal resonator can be utilized for coupling, the filter configuration is very flexible to design. In this paper, we present a SIW filter with dual-mode hexagonal cavity. By introducing coupling between source and load, the filter not only has two TZs to improve frequency selectivity, but also has a small size by profit from its single-cavity structure. 


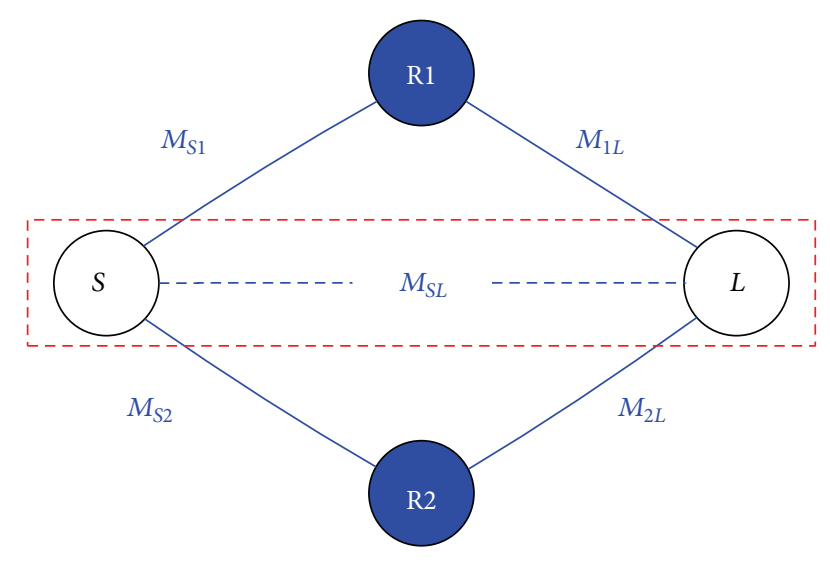

Resonator

Source/load

Figure 1: Coupling scheme of proposed dual-mode filter.

\section{Filter Analysis and Design}

Figure 1 shows the coupling topology of the proposed dualmode filter. By adding the coupling between the source and the load, one additional $T Z$ can be obtained. In other words, the source and the load are directly coupled which can add an extra transmission path. Under this circumstance, the topology can generate up to two $T Z$ s. The coupling matrix $M$ of the proposed topology can be written as

$$
M=\left[\begin{array}{cccc}
0 & M_{S 1} & M_{S 2} & M_{S L} \\
M_{S 1} & M_{11} & 0 & M_{1 L} \\
M_{S 2} & 0 & M_{22} & M_{2 L} \\
M_{S L} & M_{1 L} & M_{2 L} & 0
\end{array}\right]
$$

The conventional doublet without source-load coupling has a $T Z$ in the stopband, and an explicit expression relating the coupling elements and the transmission zero $\Omega$ is provided in a low-pass prototype as follows [11]:

$$
\Omega=\frac{M_{11} M_{S 2}^{2}-M_{22} M_{S 1}^{2}}{M_{S 1}^{2}-M_{S 2}^{2}} .
$$

Here, since the topology exhibits symmetrically, the relationships $M_{S 1}=-M_{1 L}$ and $M_{S 2}=-M_{2 L}$ can be hold.

When introducing the source-load coupling into this doublet, an additional $T Z$ can be obtained. To get more insight of location of two TZs in this topology, an explicit expression relating $M$ and the $T Z$ s is given by

$$
\Omega=a \pm\left(b^{2}+c^{2}\right)^{1 / 2},
$$

where

$$
\begin{aligned}
& a=\frac{M_{S 1}^{2}+M_{S 2}^{2}}{2 M_{S L}}-\frac{M_{11}+M_{22}}{2}, \\
& b=\frac{M_{S 2}^{2}-M_{S 1}^{2}}{2 M_{S L}}-\frac{M_{11}-M_{22}}{2},
\end{aligned}
$$

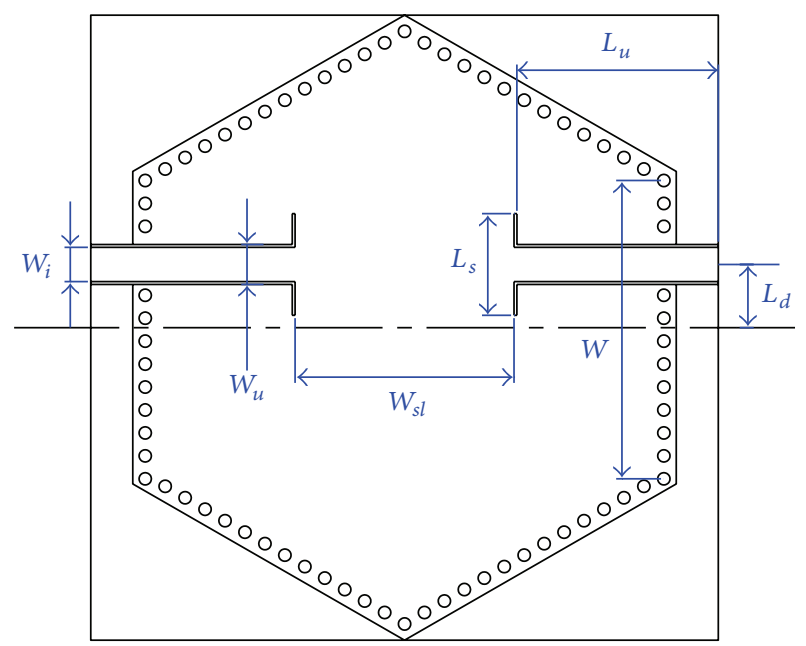

FIGURE 2: Geometric configuration of the proposed filter.

where $\Omega=\left(\omega / \omega_{0}-\omega_{0} / \omega\right) / \mathrm{FBW}$ is normalized angular frequency.

To achieve the proposed topology, a SIW filter with hexagonal dual-mode cavity is designed and embedded in a PCB substrate as shown in Figure 2. The single cavity operates with $T M_{110}$ mode which consists of two intersectant modes illustrated in Figure 3. Bypass cross-couplings between the modes and source/load are introduced through symmetrical feeding structure, while source-load coupling is introduced by the up-close input and output ports.

As far as we know, there is no exacted equation for calculating the resonant frequencies through geometrical parameters in a dual-mode hexagonal cavity. According to conventional resonant frequency formulas of metallic circular waveguide resonators, the corresponding resonant frequency of $T M_{11}$ in the hexagonal cavity can be determined by modified formulas as follows:

$$
f_{11}=\frac{C}{\sqrt{\varepsilon_{r}}} \cdot \frac{\mu^{\prime}}{2 \pi W},
$$

where $C$ is the speed of light, $\varepsilon_{r}$ is the relative dielectric constant of dielectric substrate, $\mu^{\prime}=4.27$ is the modified root coefficient based on the Bessel function, $f_{11}$ is the resonant frequencies of $T M_{11}$ mode in the hexagonal SIW cavity.

Figure 4 shows the relationship between the fitted size and the resonant frequency of the hexagonal cavity. As can be seen, the resonant frequency of $T M_{11}$ mode decreases when the geometrical parameter $W$ increases.

A feeding technique named current probe is adopted in the I/O SIW design to achieve the transition from SIW to microstrip. As the symmetrical input/output dominates the bypass cross-coupling, offset $L_{d}$ between center line and feeding structure has obvious influence on the frequency response. Figure 5 shows the frequency responses for different values of $L_{d}$. Donate the TZs at the lower and upper stopbands as $T Z_{1}$ and $T Z_{2}$, respectively. $T Z_{2}$ is produced through bypass cross coupling; thus it move towards the lower frequencies with increasing values of $L_{d}$. As $T Z_{1}$ is dominated 


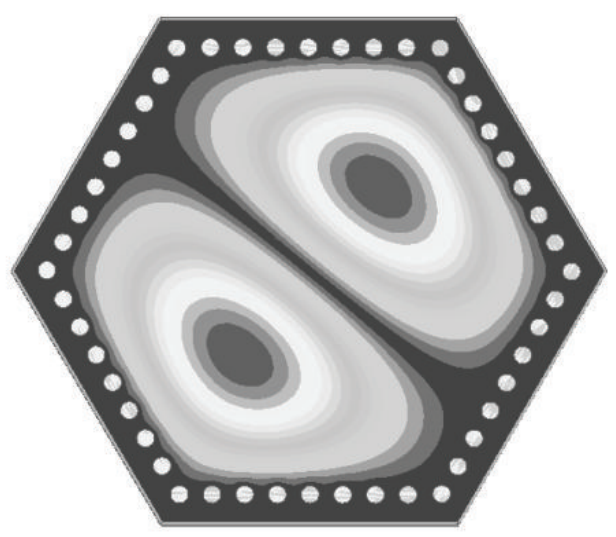

(a)

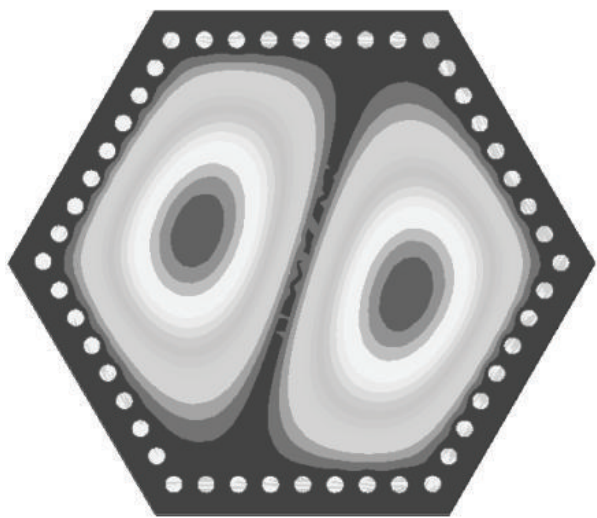

(b)

FIGURE 3: The E-field distributions of $T M_{110}$ degenerated mode: (a) left inclined mode and (b) right inclined mode.

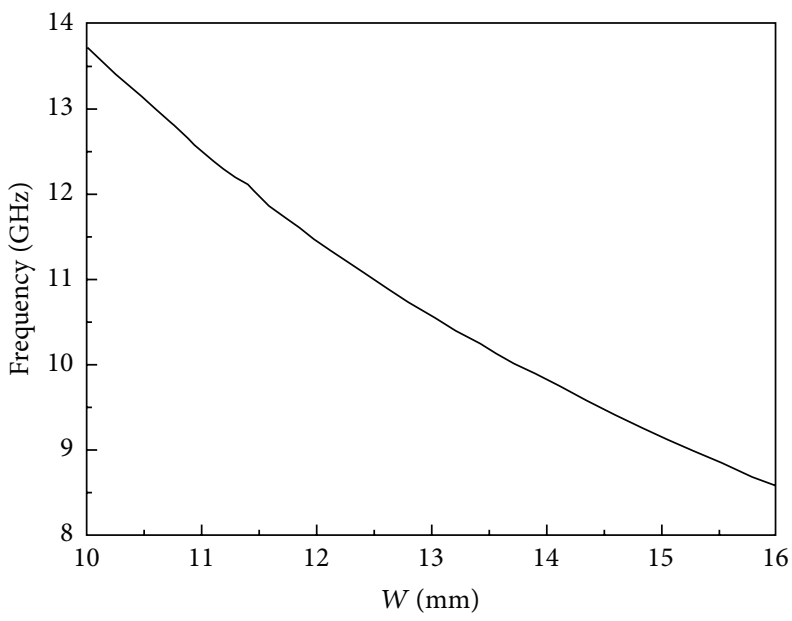

FIGURE 4: Resonant frequencies with different values of $W$.

by source-load coupling, its location changes slightly when varying the values of $L_{d}$. The feeding structure is right upon $T M_{110}$ mode, hence positions of poles change while varying $L_{d}$. As shown in Figure 5(b), $P_{1}$ and $P_{2}$ shift towards each other when the value of $L_{d}$ increases.

On the other hand, the length $\left(L_{u}\right)$ of input/output current probes in feeding structure determines not only the quality factor ( $Q$ factor) of the filter, but also the strength of source-load coupling. Frequency responses for different values of $L_{u}$ are illustrated in Figure 6. As shown in Figure 6(a), only $T Z_{2}$ at upper stopband is obtained when the value of $L_{u}$ is too small to introduce source-load coupling (e.g., $L_{u}=5.0 \mathrm{~mm}$ ). By increasing $L_{u}$ to implement source-load coupling, $T Z_{1}$ at the lower stopband can be realized. Then the increase of $L_{u}$ will result in increasing of source-load coupling; hence $T Z_{1}$ moves towards the passband. In fact, $L_{u}$ is a parameter which also influences bypass cross coupling, so $T Z_{2}$ shifts away from the center of the passband when the value of $L_{u}$ increases. $L_{u}$ has impact on $S_{11}$-parameter similar to $L_{d}$. As described in Figure 6(b), $P_{1}$ shifts away from $P_{2}$ and the passband broadens towards the lower frequencies when $L_{u}$ augments. To achieve demanded frequency responses during design process of the proposed dual-mode hexagonal SIW filter, parameter of feed probes should be carefully tuned.

\section{Experimental Results}

To validate the above-mentioned concept, a $10 \mathrm{GHz}$ hexagonal SIW filter with a $3 \mathrm{~dB}$ fractional bandwidth of $4 \%$ is fabricated on a PCB substrate with dielectric constant of 2.2. The complete parameters are finely tuned by using commercial full wave electromagnetic (EM) simulation software HFSS. Detailed dimensions of the proposed filter are illustrated in Table 1. The photograph of the fabricated filter is shown in Figure 7 . By virtue of the single hexagonal cavity and flexible source-load coupling manner, the overall size of the filter is $28.6 \mathrm{~mm} \times 28.6 \mathrm{~mm} \times 0.508 \mathrm{~mm}$.

An Agilent E8363B vector network analyzer is used for measurement. The measured and simulated frequency 


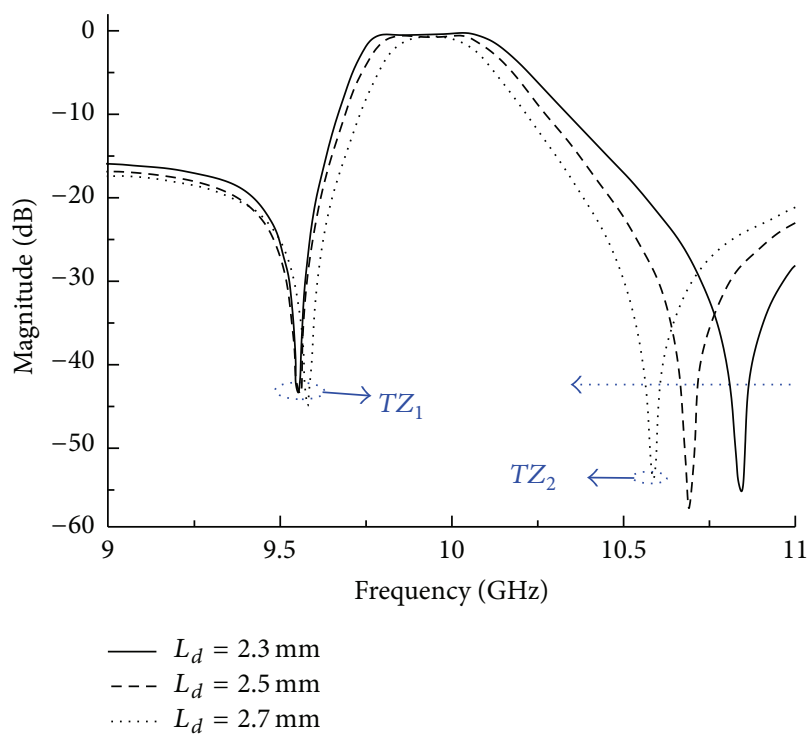

(a)

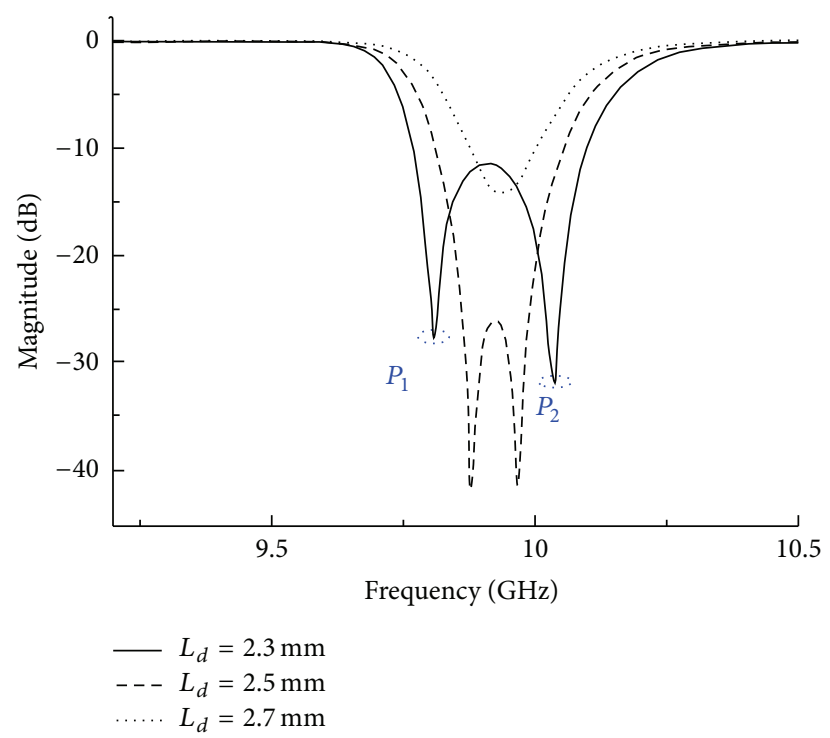

(b)

FIGURE 5: Frequency response for different values of $L_{d}$ : (a) $S_{21}$ and (b) $S_{11}$.

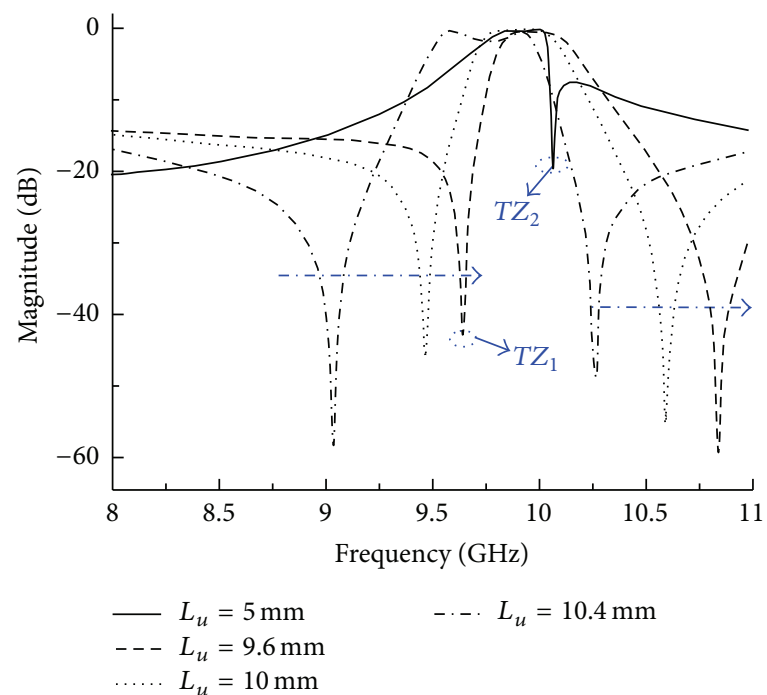

(a)

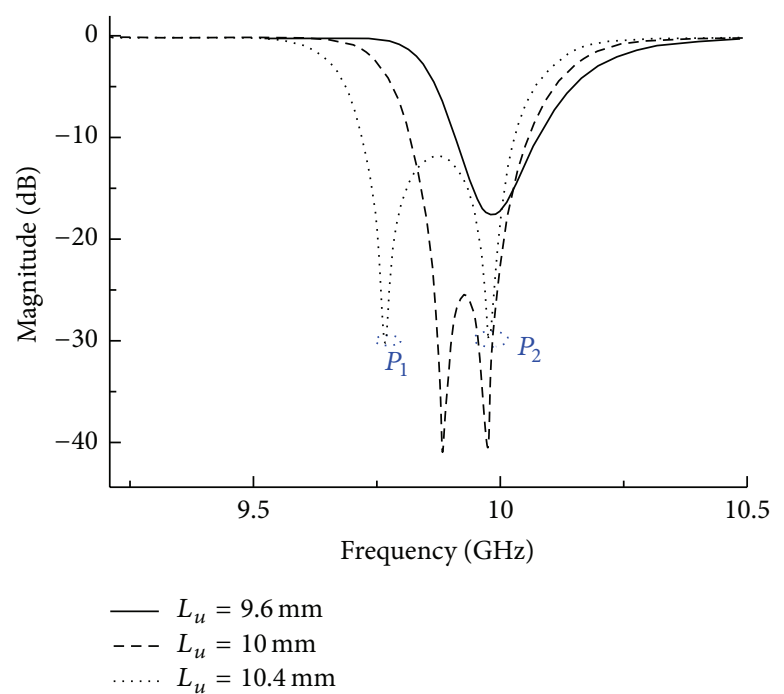

(b)

FIGURE 6: Frequency response for different values of $L_{u}$ : (a) $S_{21}$ and (b) $S_{11}$.

TABLE 1: Parameters of the proposed filter.

\begin{tabular}{lccccccc}
\hline Parameter & $W$ & $W_{i}$ & $L_{d}$ & $L_{s}$ & $W_{u}$ & $L_{u}$ & $W_{s l}$ \\
\hline Value $(\mathrm{mm})$ & 13.9 & 1.56 & 2.5 & 4.16 & 2.16 & 10.1 & 9.1
\end{tabular}

responses are plotted in Figure 8. The measured result shows a central frequency of $9.99 \mathrm{GHz}$ with a fractional bandwidth of $3.9 \%$, minimum passband insertion loss of $1.66 \mathrm{~dB}$, and inband return loss greater than $17 \mathrm{~dB}$. In addition, there are two transmission zeros located at $9.6 \mathrm{GHz}$ with $35.6 \mathrm{~dB}$ rejection and $10.75 \mathrm{GHz}$ with $42.5 \mathrm{~dB}$ rejection, respectively. The measured results are in good agreement with the simulated ones except a small frequency shift of $T Z$ s and a little discrepancy in the in-band insertion loss. The degeneration of the in-band insertion loss may be caused by the test fixture as well as the abrasion on the surface. Overall, the measured results validate the feasibility of the proposed design, with its high selectivity being demonstrated.

\section{Conclusion}

A novel compact hexagonal dual-mode SIW filter with high selectivity is proposed. Two TZs are produced to improve the frequency selectivity by introducing source-load coupling to the proposed single-cavity filter. A filter sample is fabricated, 


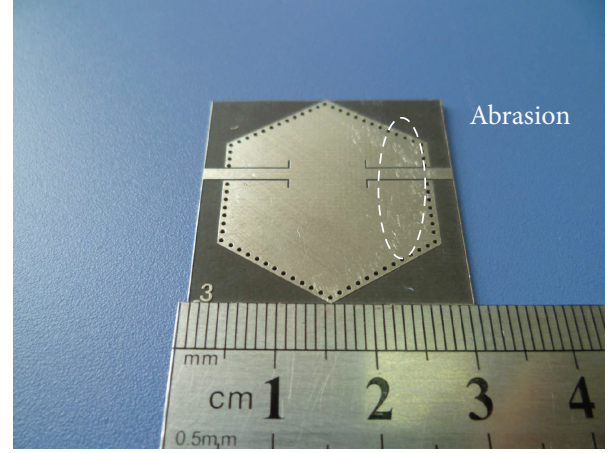

FIGURE 7: Photograph of the fabricated filter.

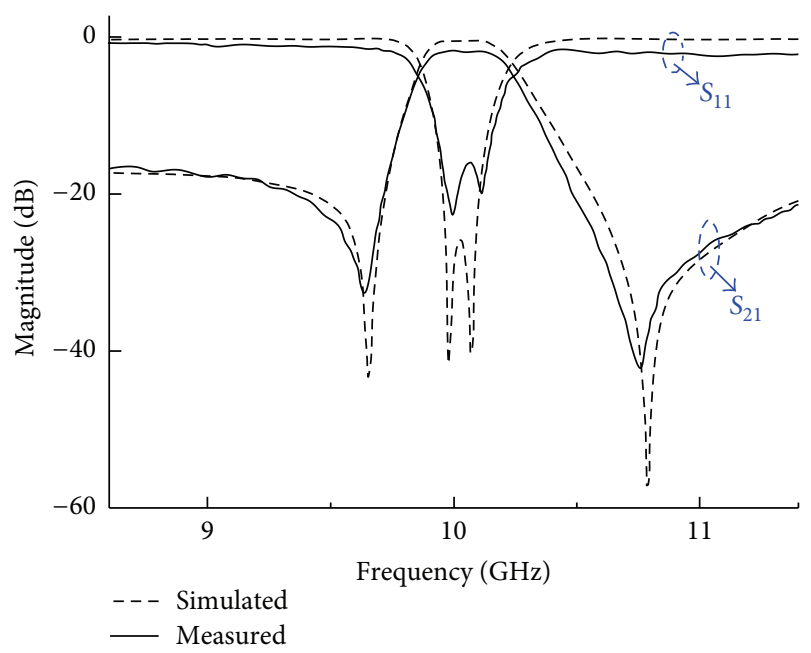

FIGURE 8: Simulated and measured results of the fabricated filter.

and the measurement results agree well with EM full wave simulation. Its compact size and high selectivity make it suitable for microwave communication application.

\section{Conflict of Interests}

The authors declare that there is no conflict of interests regarding the publication of this paper.

\section{Acknowledgments}

This work is supported by the National Natural Science Foundation of China (Grants Nos. 61101030, 61201001, and 61301052) and Fundamental Research Funds for the Central Universities of China (Grant no. ZYGX2011J132).

\section{References}

[1] W.-Y. Park and S. Lim, "Bandwidth tunable and compact bandpass filter (BPF) using complementary split ring resonators (CSRRS) on substrate integrated waveguide (SIW)," Journal of Electromagnetic Waves and Applications, vol. 24, no. 17-18, pp. 2407-2417, 2010.
[2] X.-P. Chen, K. Wu, and D. Drolet, "Substrate integrated waveguide filter with improved stopband performance for satellite ground terminal," IEEE Transactions on Microwave Theory and Techniques, vol. 57, no. 3, pp. 674-683, 2009.

[3] Z. Q. Xu, P. Wang, J. X. Liao, and Y. Shi, "Substrate integrated waveguide filter with mixed coupled modified trisections," Electronics Letters, vol. 49, no. 7, pp. 482-483, 2013.

[4] W. Y. Park and S. Lim, "Miniaturized half-mode substrate integrated waveguide bandpass filter loaded with double-sided complementary split-ring resonators," Electromagnetics, vol. 32, no. 4, pp. 200-208, 2012.

[5] W. Shen, W.-Y. Yin, and X.-W. Sun, "Miniaturized dual-band substrate integrated waveguide filter with controllable bandwidths," IEEE Microwave and Wireless Components Letters, vol. 21, no. 8, pp. 418-420, 2011.

[6] Z. Q. Xu and H. Xia, "Miniaturized multilayer dual-mode substrate integrated waveguide filter with multiple transmission zeros," Progress in Electromagnetics Research, vol. 139, pp. 627642, 2013.

[7] P.-Y. Qin, C.-H. Liang, B. Wu, and T. Su, "Novel dual-mode bandpass filter with transmission zeros using substrate integrated waveguide cavity," Journal of Electromagnetic Waves and Applications, vol. 22, no. 5-6, pp. 723-730, 2008.

[8] Y. Dong, W. Hong, H. Tang, and K. Wu, "Millimeter-wave dualmode filter using circular high-order mode cavities," Microwave and Optical Technology Letters, vol. 51, no. 7, pp. 1743-1745, 2009.

[9] W. Shen, X.-W. Sun, W.-Y. Yin, J.-F. Mao, and Q.-F. Wei, "A novel single-cavity dual mode substrate integrated waveguide filter with non-resonating node," IEEE Microwave and Wireless Components Letters, vol. 19, no. 6, pp. 368-370, 2009.

[10] Z. Q. Xu, Y. Shi, P. Wang, J. X. Liao, and X. B. Wei, "Substrate Integrated Waveguide (SIW) filter with hexagonal resonator," Journal of Electromagnetic Waves and Applications, vol. 26, no. 11-12, pp. 1521-1527, 2012.

[11] C.-K. Liao, P.-L. Chi, and C.-Y. Chang, "Microstrip realization of generalized Chebyshev filters with box-like coupling schemes," IEEE Transactions on Microwave Theory and Techniques, vol. 55, no. 1, pp. 147-153, 2007. 

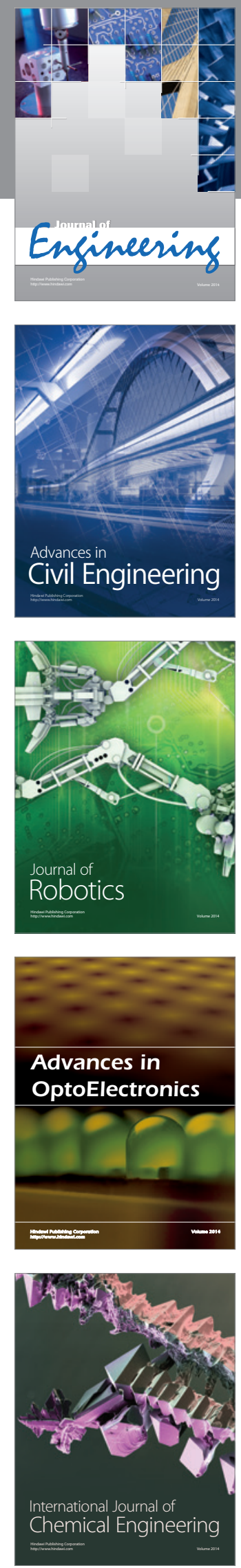

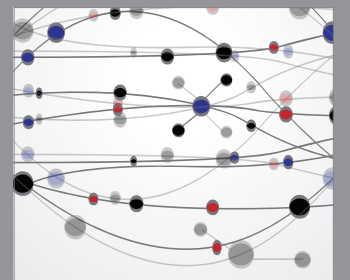

The Scientific World Journal
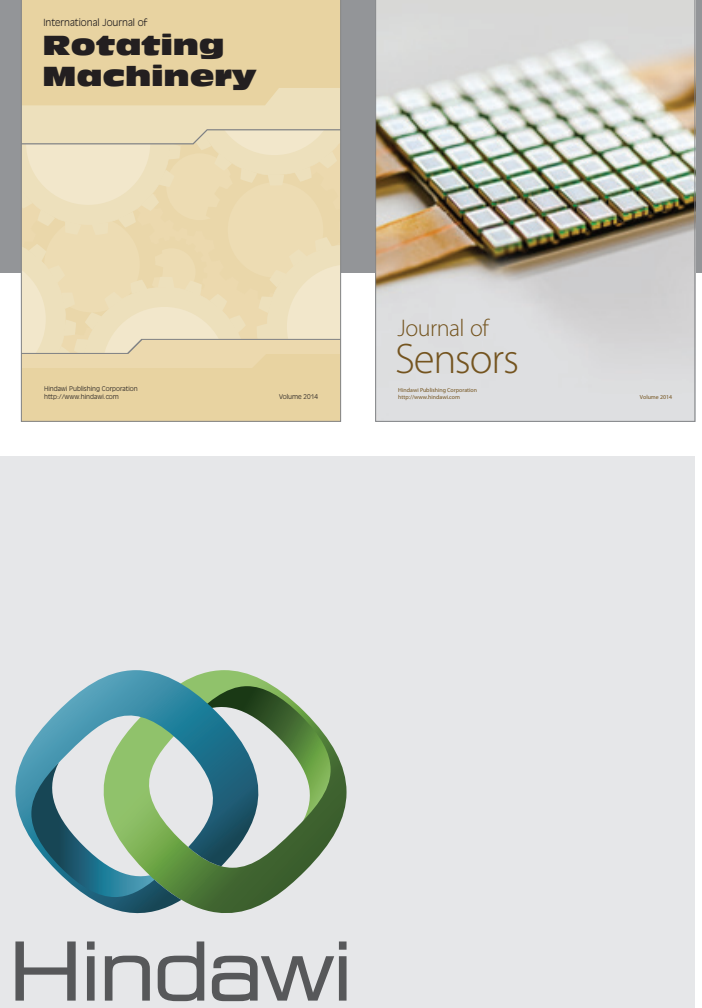

Submit your manuscripts at http://www.hindawi.com
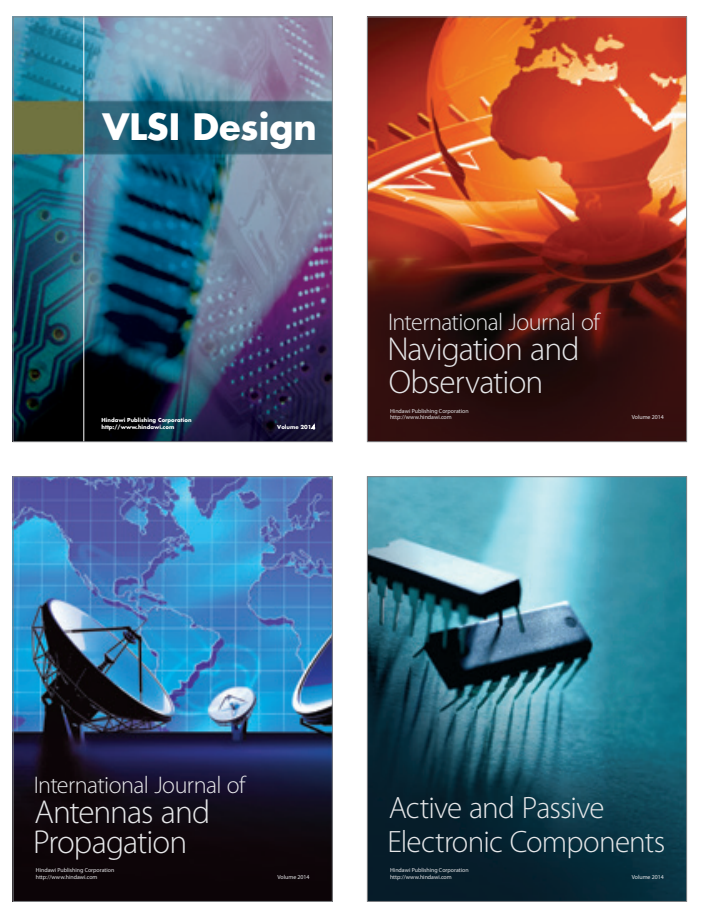
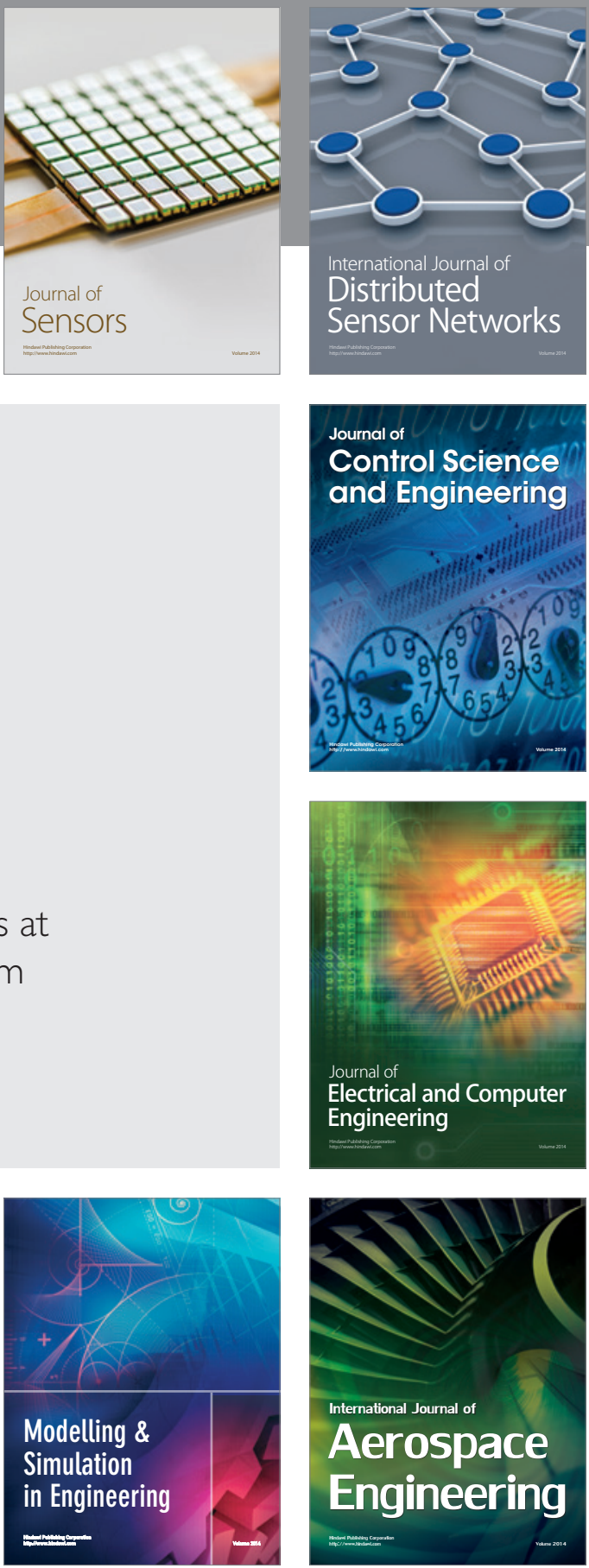

Journal of

Control Science

and Engineering
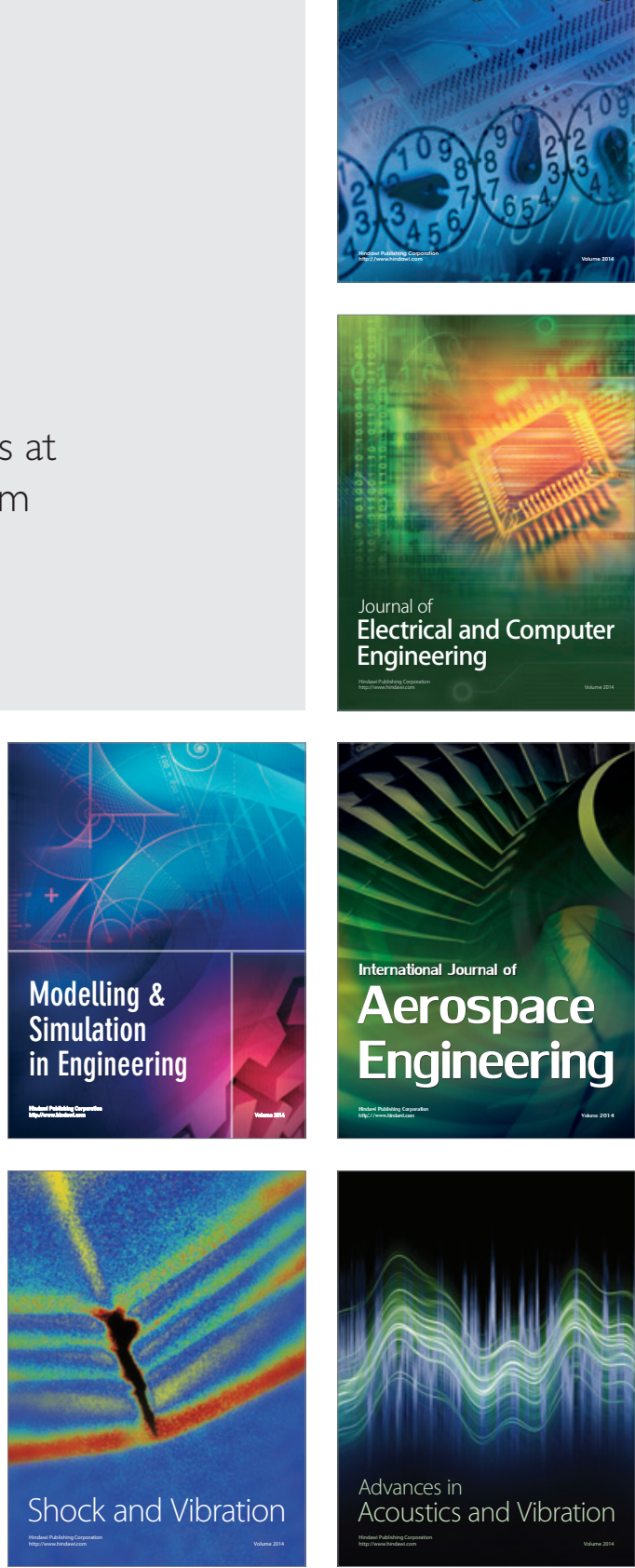